\title{
An Evaluation of Nucleated Red Blood Cell Count by an Automated Haematology Analyser- A Tertiary Centre Experience
}

\author{
Ruchee Khanna ${ }^{1}$, Deepak Nayak M. ${ }^{2}$, Seemitr Verma ${ }^{3}$, P. Lavanya ${ }^{4}$, Tanvi Shetty ${ }^{5}$, Shantha Kumari ${ }^{6}$, S. M. Zeeshan $^{7}$ \\ ${ }^{1}$ Department of Pathology, Kasturba Medical College, Manipal Academy of Higher Education, Manipal, Karnataka, \\ India. ${ }^{2}$ Department of Pathology, Kasturba Medical College, Manipal Academy of Higher Education, Manipal, \\ Karnataka, India. ${ }^{3}$ Department of Pathology, Kasturba Medical College, Manipal Academy of Higher Education, \\ Manipal, Karnataka, India. ${ }^{4}$ Department of Pathology, Kasturba Medical College, Manipal Academy of Higher \\ Education, Manipal, Karnataka, India. ${ }^{5}$ Department of Pathology, Melaka Manipal Medical College, Manipal Academy of \\ Higher Education, Manipal, Karnataka, India. ${ }^{6}$ Department of Pathology, Melaka Manipal Medical College, Manipal \\ Academy of Higher Education, Manipal, Karnataka, India. \\ ${ }^{7}$ Department of Statistics, Manipal Academy of Higher Education, Manipal, Karnataka, India.
}

\section{ABSTRACT}

\section{BACKGROUND}

nRBCs also referred to as normoblasts are seen in the peripheral blood films in variable numbers; both in physiological as well as pathologic states. Enumeration of these cells by modern day automated analysers remains a challenge. We wanted to assess the precision of the Beckman Coulter LH 755 \& 780 haematology analysers ${ }^{\mathrm{TM}}$ in enumerating the $\mathrm{nRBC}$ count.

\section{METHODS}

This is a retrospective study conducted in the Clinical Laboratory and Haematology Division of Kasturba Hospital, Manipal over a 3-month period on the Beckman Coulter LH 755 \& 780 haematology analysers ${ }^{\mathrm{TM}}$ (Beckman Coulter Inc., Miami, FL, USA) after obtaining requisite clearance from the Institutional Ethics Committee. A data of 47,332 random blood samples run on the analysers was collected. Both descriptive and analytical statistics were performed using the SPSS software version $22.0^{\mathrm{TM}}$ (Chicago, IL, USA). The sensitivity, specificity and kappa agreement were calculated using the same.

\section{RESULTS}

A total of 797 cases from the 47,332 samples showed a "flag" for nRBCs. Two Twenty of these cases were confirmed microscopically to have had nRBCs in the peripheral smear (true positives). 137 cases had nRBCs in the smear but were not definitively evaluated by the instrument (false negatives). A vast majority of the cases (577) did not reveal any nRBC on microscopic examination despite a flag generated by the machine (false positives). Additionally, a sensitivity of $27.6 \%$ and specificity of $99.7 \%$ were also noted. The kappa agreement was 0.376 which showed a fair agreement between the two methods.

\section{CONCLUSIONS}

The automated haematology analysers Beckman Coulter LH 755 \& 780 were found to be less sensitive in adequately enumerating the nucleated red blood cells. The number of false positives can be reduced by noting the 'cellular interference' flag. A manual review of such smears is necessary to confirm or refute such instrument messages.

\section{KEY WORDS}

nRBCs, Analysers, Counting, Peripheral Smear
Corresponding Author: Dr. Ruchee Khanna, Department of Pathology, Kasturba Medical College, Manipal-576104, Manipal University, Manipal, Karnataka, India. E-mail: ruchee.khanna@manipal.edu

DOI: $10.14260 /$ jemds/2020/412

Financial or Other Competing Interests: None.

How to Cite This Article:

Khanna R, Nayak DM, Verma S, et al. An evaluation of nucleated red blood cell count by an automated haematology analyser- a tertiary centre experience. J. Evolution Med. Dent. Sci. 2020;9(26): 1895-1898, DOI:
Submission 13-03-2020,

Peer Review 28-05-2020,

Acceptance 05-06-2020,

Published 29-06-2020. 


\section{BACKGROUND}

The modern-day haematology analysers have vastly improved the efficiency of the clinical laboratory. The strides in the field of cell counting have reduced the errors while increasing the reliability. Among the plethora of tests which can be reported, the complete blood count (CBC) remains the most often requested. The accurate automated enumeration and identification of platelets (PLTs), white blood cells (WBCs), and nucleated red blood cells (nRBCs) is an important and often, a challenging aspect of the complete blood count. Needless to say, a precise confirmation of these parameters by manual screening is time-consuming, expensive and the delay in reporting may adversely affect the clinical outcome of the patient. The nucleated red blood cells (nRBCs) pose a peculiar challenge in this regard.

The nRBCs also referred to as normoblasts are seen in the peripheral blood films of patients with severe haemolytic anaemia, post-splenectomy and marrow infiltration by a neoplastic process. Physiologically, small numbers may be seen in the cord blood of neonates, whereas quite large numbers are found in that of premature infants or pathologically in haemolytic disease of the new-born. Thus, a need for a correct identification and enumeration of the nRBCs, even when present in low numbers, because their presence may indicate a significant underlying disease.

The nRBCs in the context of machine counters is placed in a unique position. Due to the smaller size, an agranular cytoplasm and an eccentrically placed condensed nucleus, these infrequent cells are unpredictability included within the lymphocyte count. In scenarios where a significant numbers of nRBCs are present or if in non-lysis of red blood cells, there would be a falsely high total leucocyte count. ${ }^{1}$ Hence, a correction factor is traditionally applied to correct the total leucocyte count for the number of nRBCs by counting their percentage in the blood. ${ }^{2}$ This method suffers from low sensitivity and a statistical imprecision.

The means to circumvent this problem is where the modern-day state-of-the-art analysers have a pivotal role to play. Recent haematology analysers have the necessary software to detect and report the $\mathrm{nRBC}$ count in peripheral blood as a separate population out of WBC count. The Coulter LH 755 \& 780 haematology analysers ${ }^{\mathrm{TM}}$ (Beckman Coulter, Miami, FL, USA) are an automated system that provide a complete blood cell count on a 5-part differential WBC mode. The 5-part differential including percentage and absolute number of the leucocytes is provided by the instrument. This is achieved by the VCS technology: volume (V), using direct current, conductivity (C) using high frequency electromagnetic energy, and laser light scatter (S). ${ }^{3}$

The nRBC percentage is computed using both the WBC histogram and VCS information. This reflects the number of nRBC per 100 WBCs, since the nRBC population occupy the plot beneath the lymphocytes in VCS scatter plot. The total number of nRBCs is then calculated from the nRBC\% and the total WBC count. The WBC count is automatically corrected via a WBC interference algorithm for adjusting for interference by the particles greater than $35 \mathrm{fL} .{ }^{4}$ The presence of interfering particles in analysers often triggers a 'flag' for manual review. Although flags are helpful and result in a manual slide review, they are not highly sensitive and can often result in false positives.5,6 This implies a manual review of the sample for nRBCs, which is labour intensive and can also be imprecise. Thus, we decided to analyse the performance of the automated instrument in our laboratory with respect to nRBCs, so as to ascertain its precision.

We wanted to assess the precision of the Beckman Coulter LH 755 \& 780 haematology analysers ${ }^{\mathrm{TM}}$ in enumerating the $\mathrm{nRBC}$ count.

\section{METHODS}

The is a retrospective study (all the cases were retrieved using the request forms with attached machine report printout containing the nRBC data) conducted in the Clinical Laboratory and Haematology Division of Kasturba Hospital, Manipal over a period of 3 months (January 2015 to March 2015) after obtaining the requisite clearance from the Institutional Ethics Committee.

\section{No. of Samples}

47332 (38983 CBC counts \& 8349 peripheral smears) random samples containing $\mathrm{CBC}$ data.

\section{Instruments \& Samples}

- Beckman Coulter LH 755 \& 780 haematology analysers $^{\mathrm{TM}}$ (Beckman Coulter Inc., Miami, FL, USA) with original reagents.

- Vacutainers with EDTA-anticoagulated blood (Becton Dickinson \& Company, USA)

- Optical Microscope (Olympus Corporation, Japan)

The Coulter LH 755 \& $780^{\mathrm{TM}}$ haematology analysers under good internal quality control performed the assays within 2 hours of sample collection. To obtain the nRBC count, the blood samples were run in the $\mathrm{CBC}+\mathrm{nRBC}$ mode. In samples where a manual count of the nRBC was necessary, a peripheral smear was prepared by the manual wedge technique and stained with Leishman stain. According to the current NCCLS (Clinical and Laboratory Standards Institute) protocol, a 400-cell WBC differential count is recommended. But we decided to compare he automated results of $\mathrm{nRBC}$ with a 100-cell differential count because it reflects routine laboratory practice.

\section{Statistical Analysis}

Data was recorded on the Microsoft Excel spread sheet ${ }^{\mathrm{TM}}$. Both descriptive and analytical statistics were performed using SPSS software version 22.0 ${ }^{\mathrm{TM}}$ (Chicago, IL, USA). The sensitivity and specificity of the machine was calculated along with the kappa agreement using the same software.

\section{RESULTS}

The three month study encompassed a total of 47332 samples (including corresponding peripheral smears) run cumulatively on both the instruments. Of these, a total of 797 cases showed a 'flag' for nRBCs (Table 1). 
Of these 797 cases, 220 cases were confirmed microscopically to have had nRBCs in the peripheral smear (true positives). 137 cases had nRBCs in the smear but were not definitively evaluated by the instrument (false negatives). A vast majority of the cases (577) did not reveal any nRBC on microscopic examination despite a flag generated by the machine (false positives).

We did not compare the exact number of nRBC between the instrument and the manual count since we aimed to assess the precision of the instrument and not the accuracy.

The statistics revealed a sensitivity of $27.6 \%$ and specificity of $99.7 \%$. The kappa agreement was 0.375 which showed a fair agreement between the two methods.

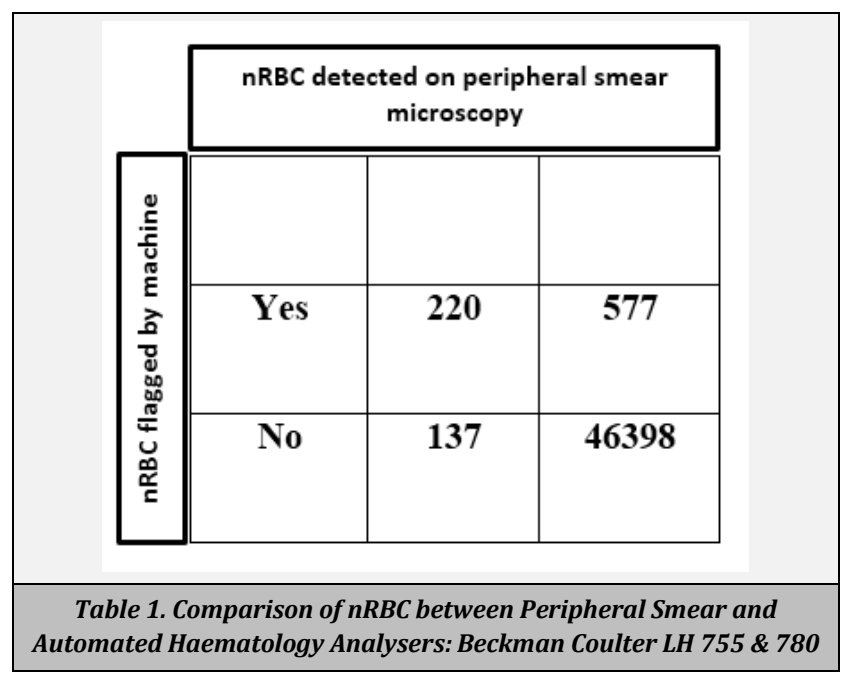

\section{DISCUSSION}

Normoblastemia is a unique term which is defined by the presence of a higher than normally expected number of nRBCs in the peripheral blood, beyond the age of approximately 1 week after full-term normal birth. ${ }^{7}$ Few of the clinical conditions which can elaborate these cells in the peripheral blood include haemoglobinopathies, haemolytic anaemias, post-splenectomy and marrow infiltration or effacement by a neoplastic condition. Thus, the nRBCs are in a sense, a window into the underlying haematologic disorder. This places a huge onus on the instruments processing the samples in a haematology laboratory, in terms of both identification and enumeration of these infrequent cells. While any number of research on nRBCs have variably affirmed or disproved the capacity of the cell counters to reliably provide an $\mathrm{nRBC}$ count $^{8-11}$, the normal daily practice still relies on a manual review of such 'flags' generated by the instrument. We use the Beckman Coulter LH 755 \& 780 haematology analysers ${ }^{\mathrm{TM}}$ (Beckman Coulter, Miami, FL, USA). Although flags for nRBCs are generated by the machine, a manual review of the smear for confirmation or refuting this message is the regular practice. Thus we decided to assess the performance of the instruments.

Due to their morphology, analysers include the nRBCs among the total WBC counts. Thus, a high $\mathrm{nRBC}$ in peripheral blood can potentially cause a falsely elevated WBC count. To counter this erroneous phenomenon, clinical laboratories have relied upon newer technologies and methods, which enable the instrument to detect, enumerate, and report nRBC counts. In some of these analysers nRBC enumeration is performed as a routine part of the automated complete blood count (CBC) with DLC, whereas in others it is performed on specifically flagged specimens in a discrete mode of a CBC $+\mathrm{nRBC}$ or CBC with DLC + nRBC. The results generated by the automated analysers include both the relative number of nRBCs per 100 WBCs (nRBC \%) and the absolute number of nRBCs per microliter of blood (nRBC\#).12 The pertinent question still remains unanswered: could the machine reliably detect the $\mathrm{nRBC}$ ?

In the present study, of the 797 cases which were flagged by the instruments as nRBCs, 220 cases were confirmed microscopically to have had nRBCs in the peripheral smear. This number is in sharp contrast to the studies reported by Aulesa $^{13}$ and Kang, ${ }^{14}$ et al who found a much higher sensitivity with the analysers ( $85 \%$ and $75 \%$ respectively). It is noteworthy that the authors in these studies included lesser number of cases.

In our study, 577 of the 797 cases had no nRBCs in the smear (false positives). This is an unusually high number given the sensitivity of the machine. In many of these cases the instrument also gave a simultaneous 'cellular interference' flag, which meant that particles other than platelets had been erroneously counted as platelets (incompletely lysed red blood cells, clumps, fibrin microclots, noise, WBC cell fragments, malarial parasites, etc.).

\section{CONCLUSIONS}

The automated haematology analysers Beckman Coulter LH $755 \& 780$ were found to be less sensitive in adequately enumerating the nucleated red blood cells. A number of false positives can be reduced by noting the 'cellular interference' flag. But a manual review of such smears is necessary to confirm or refute such instrument messages.

\section{ACKNOWLEDGEMENT}

Authors thank the officials at the Medical Records Department, Kasturba Hospital, Manipal, for the timely cooperation and support.

\section{REFERENCES}

[1] Bain BJ. Blood cells: a practical guide. $4^{\text {th }}$ edn. Massachusetts: Blackwell Publishing 2006: p. 175-97.

[2] Bain BJ. Blood cell morphology in health and disease. In: Lewis SM, Bain BJ, Bates I, eds. Dacie and Lewis Practical haematology. 10 $10^{\text {th }}$ edn. Philadelphia: Churchill Livingstone/ Elsevier 2006: p. 79-113.

[3] Beckman Coulter. Coulter LH750 system: Operation principles. Operator's Guide. 2004:9.

[4] Coulter Corporation. Coulter LH 755 System. Reference manual. Miami: A Beckman Coulter Company, 2002. 
[5] Brown W, Kaplan SS, Keeney M, et al. Workflow improvement with random access and enhanced flagging on the new Coulter LH 750 haematology analyser. Laboratory haematology 2001;7(4):229-35.

[6] Yee IC, Keeney M, Johnson K, et al. White blood cell flagging rates of the coulter LH 750 analyser compared with the Coulter Gen-S haematology analyser. Laboratory haematology 2001;7:211-6.

[7] Gulati G, Behling E, Kochar W, et al. An evaluation of the performance of Sysmex XE-2100 in enumerating nucleated red cells in peripheral blood. Arch Pathol Lab Med 2007;131(7):1077-83.

[8] Fernandez T, Domack LB, Montes D, et al. Performanc evaluation of the coulter LH 750 haematology analyser. Lab haematol 2001;7:217-28.

[9] Ghys T, Malfait R, Van den Bossche J. Performance evaluation of the Sysmex XS-1000i automated haematology analyser. Int $\mathrm{J}$ Lab haematol 2009;31(5):560-6.
[10] Tsuji T, Sakata T, Hamaguchi Y, et al. New rapid flow cytometric method for the enumeration of nucleated red blood cells. Cytometry 1999;37(4):291-301.

[11] Igout J, Fretigny M, Vasse M, et al. Evaluation of the Coulter LH 750 haematology analyser compared with flow cytometry as the reference method for WBC, platelet and nucleated RBC count. Clin Lab Haem 2004;26(1):1-7.

[12] De Keijer MH, van Der Meer W. Automated counting of nucleated red blood cells in blood samples of newborns. Clin Lab Haem 2002;24(6):343-5.

[13] Aulesa C, Pastor I, Naranjo D, et al. Validation of the Coulter LH 750 in a hospital reference laboratory. Lab haematol 2003;9(1):15-28.

[14] Kang SH, Kim HK, Ham CK, et al. Comparison of four haematology analysers, CELL-DYN Sapphire, ADVIA 120, Coulter LH 750 and Sysmex XE-2100, in terms of clinical usefulness. Int J Lab haematol 2008;30(6):480-6. 